\title{
Carbon C 14 Roniciclib
}

National Cancer Institute

\section{Source}

National Cancer Institute. Carbon C 14 Roniciclib. NCI Thesaurus. Code C121551.

An orally bioavailable radioconjug ate composed of roniciclib, a cyclin dependent kinase (CDK) inhibitor, radiolabeled with carbon C 14, with potential use for evaluating the pharmacokinetic profile of roniciclib. Roniciclib selectively binds to and inhibits the activity of various CDK subtypes, which leads to cell cycle arrest and an inhibition of tumor cell proliferation. Labeling of roniciclib with the radioactive tracer carbon C 14 allows for the evaluation of roniciclib's pharmacokinetic profile, including its absorption, distribution, metabolism, and excretion (ADME). CDKs, serine/threonine kinases overexpressed in various tumor cell types, play key roles in the regulation of both cell cycle prog ression and cellular proliferation. 